\title{
Técnicas endoscópicas para bariatría
}

\author{
Juan C. Caramés-Aranda* y Minerva Cárdenas \\ Centro de Endoscopia Terapéutica Avanzada de Reynosa, Reynosa, Tamaulipas, México
}

La obesidad es en la actualidad un problema de salud, considerado incluso de dimensiones pandémicas. Actualmente, tan solo en los EE.UU., más de 108 millones de adultos tienen un índice de masa corporal (IMC) mayor de $30 \mathrm{~kg} / \mathrm{m}^{2}$. Un estimado de 24 millones de estadounidenses adultos tiene obesidad severa, con IMC mayor de $40 \mathrm{~kg} / \mathrm{m}^{2}$ que podrían calificar para Cirugía Bariátrica. Sin embargo, menos del $1 \%$ de ellos reciben tratamiento quirúrgico de obesidad, lo que significa alrededor de 213,200 personas por año $0^{1,2}$. Aproximadamente 84 millones de personas presentan enfermedad grasa del hígado no alcohólica (NAFLD), 88 millones de habitantes son pre diabéticos y alrededor de 34 millones son diabéticos, condicionando un gasto en salud muy elevado.

La carga global de la obesidad y sus comorbilidades asociadas han originado la necesidad urgente de opciones de tratamiento adicionales para pelear contra esta pandemia. Las diferentes terapias endoscópicas bariátricas (TEB) proveen un tratamiento mínimamente invasivo efectivo contra la obesidad que incrementan las opciones más allá de la cirugía bariátrica, tratamiento médico y cambios en el estilo de vida.

En la semana de enfermedades digestivas (DDW) 2021, debido a la seguridad de los procedimientos bariátricos endoscópicos, se recomienda tratar endoscópicamente a los pacientes con IMC $>30 \mathrm{~kg} / \mathrm{m}^{2} \sin$ comorbilidades o pacientes con IMC $\geq$ de $27 \mathrm{~kg} / \mathrm{m}^{2}$ con una o más comorbilidades ${ }^{3,4}$.

Dentro de las TEB disponibles existen procedimientos dirigidos al estómago y otros al intestino delgado, cada una con diferentes modalidades. Las diferentes TEB dirigidas al estómago logran la reducción de peso por diversas vías como son: ocupando espacio, remodelando la anatomía y el tamaño, obstruyendo el vaciamiento y finalmente el sistema de aspiración. Los métodos que ocupan espacio son los balones intragástricos, de los cuales se han desarrollado diferentes modelos aprobados por la FDA, sin embargo, los que han reportado mejores resultados son el Spatz III y el Orbera, con más del $10 \%$ pérdida del peso corporal total (TBWL) y mejoría en las comorbilidades ${ }^{5}$. El factor más importante del mecanismo de acción de los balones es el retraso en el vaciamiento gástrico que se logra de manera más efectiva con los balones llenos con líquido comparado con los balones llenos con aire $^{6}$.

Dentro de los TBE que remodelan el estómago se encuentran la manga gástrica endoscópica (MGE) y el POSE 2.0. Ambos procedimientos tienen alta aceptación, son bien tolerados, sus resultados son efectivos, más duraderos comparados con los balones y siguen el concepto de la cirugía bariátrica, con la ventaja de que no realizamos heridas en la piel del abdomen y que ambos pueden ser procedimientos ambulatorios, con una rápida recuperación.

La manga gástrica endoscópica modifica la longitud y el diámetro del estómago, alterando así la acomodación y el efecto de saciedad. Se mencionó un metaanálisis que incluyó 1,772 pacientes logando un $17 \%$ del TBWL a los 12 meses, con solo un $2 \%$ de incidencia de eventos adversos ${ }^{4}$. En otro estudio realizado por 47 endoscopistas brasileños con 1,828 pacientes, lograron un $18.2 \%$ de pérdida del TBWL a los 12 meses, con solo $0.8 \%$ de eventos adversos. En este estudio se describió que el IMC ideal para la manga gástrica

\section{Correspondencia:}


endoscópica es de $30-35 \mathrm{~kg} / \mathrm{m}^{2}$, sin límite superior o edad de inclusión ${ }^{7}$. La técnica de POSE 2.0 utiliza una plicatura cada $7 \mathrm{~cm}$ en la curvatura mayor, iniciando sobre la incisura angularis y extendiéndose hasta el cardias, sin modificar el fondo. Se realizan un total de 20 plicaturas con un patrón específico, logrando así la disminución de la longitud y del diámetro axial del estómago, alterando la motilidad y el vaciamiento ${ }^{8}$.

Se presentó un trabajo en el que se evaluó la eficacia de la MGE en paciente con obesidad grado III (IMC $\geq$ $40 \mathrm{~kg} / \mathrm{m}^{2}$ ), en términos de pérdida de peso y disminución de comorbilidades, en pacientes que no fueron candidatos a cirugía o que no aceptaron la cirugía, con un seguimiento mínimo de 12 meses. Se logró disminuir el IMC del promedio de $43.9 \mathrm{~kg} / \mathrm{m}^{2}$ a $34.8 \mathrm{~kg} / \mathrm{m}^{2}$, con un $20 \%$ de $\mathrm{TWL}$, logrando impactar de manera significativa en las comorbilidades como hipertensión arterial, diabetes mellitus, hiperinsulinemia y el síndrome de apnea del sueño obstructiva ${ }^{9}$. Concluyeron que la MGE tiene buenos resultados en términos de pérdida de peso y disminución de comorbilidades, pero que no logra resolver la obesidad, sin embargo, es un procedimiento que se puede repetir (REDO) y debe ser considerada como una alternativa válida y menos invasiva cuando la cirugía no sea posible.

En otro estudio del grupo Dr. Michel Kahaleh, se revisó la evolución a largo plazo y la necesidad de revisión de la MGE. El objetivo principal fue evaluar los factores del paciente y de la técnica que predicen la reganancia de peso y la necesidad de rehacer la MGE. De un total de 51 pacientes incluidos en un periodo de 28 meses, todos con IMC > $43 \mathrm{~kg} / \mathrm{m}^{2}$, solo 11 pacientes (21.56\%) necesitaron revisión de la MGE ya sea por reganancia de peso $(n=5)$ o por que querían perder más peso $(n=6)$. Los pacientes con mayor número de suturas se asociaron a menor necesidad de rehacer la $M^{\prime} E^{10}$.

De los métodos endoscópicos dirigidos al intestino delgado, se comentaron resultados satisfactorios con mejoría de factores metabólicos, más que en la pérdida de peso. De los TEB en duodeno existen los dispositivos de bypass que evitan la absorción de nutrientes en los primeros $65 \mathrm{~cm}$ de longitud del yeyuno proximal.

Las técnicas de ablación utilizan el mismo principio que para la ablación del esófago de Barrett, inyectando solución salina en el espacio submucoso y aplicando ablación térmica a $90{ }^{\circ} \mathrm{C}$, durante 10 segundos, en un área de $10 \mathrm{~cm}$ de longitud para regenerar la mucosa duodenal. Finalmente se comentaron los métodos de desviación biliar y de anastomosis endoscópicas utilizando magnetos para crear una anastomosis entre el duodeno y el yeyuno, con resultados favorables a nivel metabólico, revirtiendo la resistencia a la insulina. En conclusión, las terapias endoscópicas bariátricas juegan un papel muy importante en el manejo de la obesidad y sus complicaciones metabólicas.

\section{Financiamiento}

La presente investigación no ha recibido ayudas específicas provenientes de agencias del sector público, sector comercial o entidades sin ánimo de lucro.

\section{Conflicto de intereses}

Los autores declaran que no hay conflicto de intereses.

\section{Bibliografía}

1. Fryar CD, Carroll MD, Afful J. Prevalence of overweight, obesity, and severe obesity among adults aged 20 and over; United States, 1960-1962 trought 2017-2018 [Internet]. EE.UU.: Centers for Disease Control and Prevention, National Center for Health Statistics; 29 de enero de 2021. Disponible en: https://www.cdc.gov/nchs/data/hestat/obesity-adult-17-18/ obesity-adult.htm

2. Petersen MP. American Diabetes Association. Diabetes Care. 2018;41:917-28.

3. ASGE Bariatric Endoscopy Task Force and ASGE Technology Committee; Dayyeh BKA, Kumar N, Edmundowicz SA, Jonnalagadda S, Larsen M, Sullivan S, et al. ASGE Bariatric Endoscopy Task Force systematic review and meta-analysis assesing the ASGE PIVI threholds for adopting endoscopic bariatric therapies. Gastrointest Endosc. 2015;82(3):425-38.

4. Hedjoudje A, Abu Dayyeh BK, Cheskin LJ, Adam A, Neto MG, Badurdeen D, et al. Efficacy and safety of endoscopic sleeve gastroplasty: A systematic review and meta-analysis. Clin Gastroenterol Hepatol. 2020;18:1043-53.

5. Abu Dayyeh BK, Edmundowicz S, Thompson CC. Clinical Practice Update: Expert review on endoscopic bariatric therapies. Gastroenterology. 2017; 152:716-29.

6. Vargas EJ, Bazerbachi F, Calderon G, Prokop LJ, Gomez V, Murad MH, et al. Changes in time of gastric emptying after surgical and endoscopic bariatrics and weight loss: A systematic review and meta-analysis. Clin Gastroenterol Hepatol. 2020;18(1):57-68.e5.

7. Galvao Neto M, Bezerra Silva L, de Quadros LG, Grecco E, Concon Filho A, Menezes Barbosa de Amorim A, et al. Brazilian Consensus on Endoscopic Sleeve Gastroplasty. Obes Surg. 2021;31(1):70-8.

8. Lopez-Nava G, Asokkumar R, Turró Arau R, Neto MG, Dayyeh BA. Modified primary obesity surgery endoluminal (POSE-2) procedure for the treatment of obesity. VideoGIE. 2020;5(3):91-3.

9. Pontecorvi V, Laterza L, Bove V, Carlino G, Gallo C, Orlandini B, et al. ID: 3525747 Short-term outcomes of endoscopic sleeve gastroplasty in type III obese patients: a clinical, retrospective, single center study. Gastrointest Endosc [Internet]. 2021;93(6 Suppl):AB7-AB8. Disponible en: https://www.giejournal.org/article/S0016-5107(21)00327-8/fulltext

10. Gjeorgejievski M, Bhurwal A, Abdelqader A, Chouthai AA, Chen AG, Liao K, et al. ID: 3523424 Endoscopic sleeve gastroplasty successes and failures: predictors for ESG revision. Gastrointest Endosc [Internet]. 2021;93(6 Suppl):AB5. Disponible en: https://www.giejournal.org/article/ S0016-5107(21)00322-9/abstract 